OPEN ACCESS

Edited by:

Pascale Alard,

University of Louisville, USA

Reviewed by:

Fernando Navarro-Garcia,

Center for Advanced Research, The National Polytechnic Institute,

Cinvestav-IPN, Mexico

Mauricio J. Farfan,

Universidad de Chile, Chile

${ }^{*}$ Correspondence:

Josée Hare

josee.hare/@umontreal.ca

Grégory Jubelin

gregory.jubelin@inra.fr

Received: 27 January 2017 Accepted: 31 March 2017

Published: 24 April 2017

Citation:

Le Bihan G, Sicard J-F, Garneau P, Bernalier-Donadille A, Gobert AP, Garrivier A, Martin C, Hay AG,

Beaudry $F$, Harel $J$ and Jubelin $G$

(2017) The NAG Sensor NagC Regulates LEE Gene Expression and Contributes to Gut Colonization by Escherichia coli 0157:H7.

Front. Cell. Infect. Microbiol. 7:134. doi: $10.3389 /$ fcimb.2017.00134

\title{
The NAG Sensor NagC Regulates LEE Gene Expression and Contributes to Gut Colonization by Escherichia coli 0157:H7
}

\begin{abstract}
Guillaume Le Bihan ${ }^{1}$, Jean-Félix Sicard ${ }^{1}$, Philippe Garneau ${ }^{1}$, Annick Bernalier-Donadille ${ }^{2}$, Alain P. Gobert ${ }^{2}$, Annie Garrivier ${ }^{2}$, Christine Martin ${ }^{2}$, Anthony G. Hay ${ }^{3}$, Francis Beaudry ${ }^{4}$, Josée Harel ${ }^{1 *}$ and Grégory Jubelin ${ }^{2 *}$

${ }^{1}$ Faculté de Médecine Vétérinaire, Centre de Recherche en Infectiologie Porcine et Aviaire, Université de Montréal, Saint-Hyacinthe, QC, Canada, ${ }^{2}$ INRA, Université Clermont Auvergne, MEDIS, Clermont-Ferrand, France, ${ }^{3}$ Department of Microbiology, Cornell University, Ithaca, NY, USA, ${ }^{4}$ Groupe de Recherche en Pharmacologie Animal du Québec, Département de Biomédecine Vétérinaire, Faculté de Médecine Vétérinaire, Université de Montréal, Saint-Hyacinthe, QC, Canada
\end{abstract}

Enterohemorrhagic Escherichia coli (EHEC) O157:H7 are human pathogens responsible for bloody diarrhea and renal failures. EHEC employ a type 3 secretion system to attach directly to the human colonic epithelium. This structure is encoded by the locus of enterocyte effacement (LEE) whose expression is regulated in response to specific nutrients. In this study, we show that the mucin-derived sugars $\mathrm{N}$-acetylglucosamine (NAG) and N-acetylneuraminic acid (NANA) inhibit EHEC adhesion to epithelial cells through down-regulation of LEE expression. The effect of NAG and NANA is dependent on NagC, a transcriptional repressor of the NAG catabolism in E. coli. We show that $\mathrm{NagC}$ is an activator of the LEE1 operon and a critical regulator for the colonization of mice intestine by EHEC. Finally, we demonstrate that NAG and NANA as well as the metabolic activity of Bacteroides thetaiotaomicron affect the in vivo fitness of EHEC in a NagC-dependent manner. This study highlights the role of NagC in coordinating metabolism and LEE expression in EHEC and in promoting EHEC colonization in vivo.

Keywords: NagC, LEE, EHEC, $\mathrm{N}$-acetylglucosamine (or eventually NAG), $\mathrm{N}$-acetylneuraminic acid (or eventually NANA)

\section{INTRODUCTION}

Escherichia coli O157:H7 are human foodborne pathogens responsible for outbreaks mostly in developed countries. Infections by EHEC occur following ingestion of contaminated food and provoke symptoms ranging from watery or bloody diarrhea to hemolytic and uremic syndrome (HUS). A range of virulence factors are involved in EHEC O157:H7 pathogenicity including the Shiga-toxin which is associated with development of HUS, and the T3SS which enables the pathogen to attach to the intestinal epithelium and cause diarrhea (Kaper et al., 2004).

T3SS-encoding genes are gathered into the locus of enterocyte effacement (LEE) that is composed of five operons (LEE1 to LEE5) which encode for structural proteins, regulators, chaperones and effectors that are secreted into the host cells (Kaper et al., 2004). The first gene of the LEE, ler, encodes an activator that regulates the five major LEE operons. Expression of 
ler is controlled by several regulators in response to intestinal metabolites, such as bacterial waste products (Nakanishi et al., 2009), quorum-sensing molecules (Sircili et al., 2004), hormones (Walters and Sperandio, 2006), biotin (Yang et al., 2015), fucose (Pacheco et al., 2012), and ethanolamine (Kendall et al., 2012).

During its infectious cycle, EHEC O157:H7 encounters a large amount of mucin-derived sugars (Fabich et al., 2008; Bertin et al., 2013). Mucin is part of the mucous layer covering the intestinal epithelium and is heavily O-glycosylated. The mucous layer is a physical barrier that limits contact between bacteria and host epithelial cells (McGuckin et al., 2011). By producing specific glycosidases, several species of the gut microbiota release sugars from O-glycans into the intestinal lumen (Bertin et al., 2013; $\mathrm{Ng}$ et al., 2013; Elhenawy et al., 2014). Released mucin sugars, including $\mathrm{N}$-acetylglucosamine (NAG), $\mathrm{N}$-acetylneuraminic acid (NANA), galactose, fucose, mannose and $\mathrm{N}$-acetylgalactosamine, represent an important reservoir of nutrients that promotes the growth of commensal and pathogenic bacteria including E. coli (Fabich et al., 2008; Bertin et al., 2013; Conway and Cohen, 2015). Escherichia coli and more particularly EHEC O157:H7 are able to concomitantly metabolize in vitro up to nine mucin sugars at a time, and preferentially use NAG and galactose (Fabich et al., 2008; Bertin et al., 2013; Conway and Cohen, 2015).

Genes involved in the catabolism of sugars are often regulated by proteins responding to the presence of their cognate sugar. For example, the regulator $\mathrm{NagC}$, known as a repressor of NAG and galactose catabolism, is a NAG-6 phosphate (NAG-6P) sensing protein, NAG-6P being produced during the catabolism of NAG and NANA (Plumbridge, 1991; El Qaidi et al., 2009). When NAG-6P concentrations are low, NagC acts as a DNA binding protein, an activity that is lost with high intracellular NAG-6P concentration (Plumbridge and Kolb, 1991; Sohanpal et al., 2004). In addition to the role they play as nutrients, some mucin sugars can act as regulatory signals that influence bacterial colonization and adherence to cells (Sohanpal et al., 2004; Barnhart et al., 2006; Pacheco et al., 2012).

Previously, we have shown that EHEC O157:H7 respond to the metabolic activity of the human gut microbiota by activating the expression of genes required for NANA utilization and by down-regulating the expression of the LEE genes (Le Bihan et al., 2015). In this study, the effect of NANA and NAG on the adhesion phenotype of EHEC O157:H7 was examined. We found that NANA and NAG are inhibitors of EHEC O157:H7 adhesion to epithelial cells. We demonstrated that NANA and NAG reduce the expression of the five LEE operons in a NagCdependent way. Mutation in nagC diminished the expression of LEE genes. In addition, NagC was shown to bind directly to the LEE1 promoter region, thereby could influence expression of ler gene, which encodes the LEE master regulator. We also show that NagC promotes EHEC colonization of mouse intestine. Further, we demonstrate that exogenous addition of NAG into the intestine or gavage with the mucin degrading commensal Bacteroides thetaiotaomicron modulates the fitness of EHEC in vivo in a NagC-dependent manner. Taken together, our data indicate that NagC coordinates the catabolism of mucus-derived sugars and T3SS production, and promotes EHEC intestinal colonization.

\section{MATERIALS AND METHODS}

\section{Bacteria, Mutagenesis, and Growth Conditions}

Strains and plasmids are listed in Table EV1. The EHEC O157:H7 strain EDL933 (O'Brien et al., 1983) was used in this study. B. thetaiotamicron strain VPI-5482 was grown anaerobically at $37^{\circ} \mathrm{C}$ in a complex medium containing clarified rumen fluid (Leedle and Hespell, 1980). The medium was prepared, dispensed and inoculated by using strictly anaerobic techniques in Balch tubes. The EDL933 $\triangle n a g C$ and $\triangle n a n R$ mutants were generated by allelic exchange using a suicide vector as described in EV Methods. When required, the growth medium was supplemented with kanamycin $(25 \mathrm{mg} / \mathrm{ml})$, ampicillin $(50 \mathrm{mg} / \mathrm{ml})$, NANA (0.1 mM or $1 \mathrm{mM})$, or NAG (0.1 mM or $1 \mathrm{mM})$ (Sigma Aldrich).

\section{Beta-Galactosidase Assays}

The entire intergenic region between ler (LEE1) and espG (bp $-1,225$ to +19 ) containing two ler promoters (Sperandio et al., 2002; Porter et al., 2005) was inserted upstream of lacZ in pRS551 (see EV Methods). The resulting plasmid pGLB was introduced into EDL933 or its isogenic mutants. After growth in DMEM with or without NANA or NAG until $\mathrm{OD}_{600}$ of 0.6 , $\beta$-galactosidase assays were performed as described previously (Miller, 1972). Student $t$-tests were performed to determine statistical significance.

\section{Quantitative Real Time PCR (qRT-PCR)}

Bacteria were harvested at $\mathrm{OD}_{600}$ of 0.6 and RNA was extracted as previously described (Le Bihan et al., 2015). cDNAs were synthesized from $10 \mu \mathrm{g}$ RNA using reverse transcriptase. The concentration of cDNA samples was then adjusted to $25 \mathrm{ng} / \mu \mathrm{L}$. A standard curve was performed for genes of interest to determine the copy number of targeted transcripts in $50 \mathrm{ng}$ of cDNA. Primers used in this study are listed in Table EV2. Results are presented as the ratios between the cDNA copy number of the gene of interest and the cDNA copy number of the housekeeping gene. Student $t$-tests were performed to calculate $p$-values.

\section{Western Blotting}

Western blot analyses were performed with slight modifications to those previously described (Chekabab et al., 2014). Culture supernatants $(8 \mathrm{ml})$ were harvested and supplemented with $1 \mu \mathrm{g}$ Bovine Serum Albumin (BSA). Proteins were precipitated overnight at $4^{\circ} \mathrm{C}$ using $10 \%$ trichloroacetic acid and sodium deoxycholate, pelleted by centrifugation, washed with acetone, resuspended in SDS sample buffer and boiled. Proteins were then run on $14 \%$ SDS-PAGE gels and transferred to nitrocellulose membranes. Protein transfer including BSA was assessed using Ponceau $S$ dye. The EspB protein was revealed using a rabbit EspB specific polyclonal antiserum $(1: 2,000)$ and a goat antirabbit HRP-linked secondary antibody (Bio-Rad Laboratories, Hercules, CA).

\section{Cell Culture and Bacterial Infections}

Epithelial cell lines HeLa, HCT-8, and HCT-116 were maintained in MEM with $10 \% \mathrm{FBS}, 100 \mathrm{U} / \mathrm{mL}$ penicillin and $100 \mathrm{mg} / \mathrm{mL}$ 
streptomycin at $37^{\circ} \mathrm{C}$ under $5 \% \mathrm{CO}_{2}$ (Branchu et al., 2014). Cells were seeded into 6 -well plates $\left(5 \times 10^{5}\right.$ cells/well $)$ and grown for $24 \mathrm{~h}$ without antibiotics. Bacteria were pre-grown in DMEM in the presence or absence of NANA or NAG before the infection assays. Cells were then washed and infected with bacteria with an MOI of 10 for $90 \mathrm{~min}$, in the presence or absence of NANA or NAG. Following infection, cells were washed 3 times with DPBS, trypsinized for $5 \mathrm{~min}$ at $37^{\circ} \mathrm{C}$, pelleted by a low-speed centrifugation $(100 \mathrm{~g} ; 3 \mathrm{~min})$. The pellet was washed once to ensure the plating of adherent bacteria only. Results are presented as the percentage of adherent bacteria as compared to the wildtype strain incubated without NANA or NAG. The EDL933 $\triangle e s c N$ strain that does not produce the T3SS (Deng et al., 2004) was used as a negative control. Student $t$-tests were performed to determine the significance.

\section{Electrophoretic Mobility Shift Assays (EMSA)}

The EMSA was adapted from previous report (Chekabab et al., 2014). The EMSA reaction mix consisted of purified NagC at the desired concentration $(0.5-2.5 \mu \mathrm{M}), 50 \mathrm{nM}$ of $5^{\prime}$ 6-FAM labeled pLEE1 probe, $0.1 \mathrm{mg} / \mathrm{mL}$ calf thymus DNA and $0.1 \mathrm{mg} / \mathrm{mL} \mathrm{BSA}$ in EMSA buffer $(50 \mathrm{mM} \mathrm{NaCl}, 20 \mathrm{mM}$ Tris, $\mathrm{pH} 7.4,0.02 \% \mathrm{v} / \mathrm{v}$ sodium azide). Reactions were incubated for $30 \mathrm{~min}$ at $25^{\circ} \mathrm{C}$, and then loaded onto a $12 \%$ native polyacrylamide gel running at $120 \mathrm{~V}$ in $1 \mathrm{x}$ TBE buffer. The forward primer contained a $5^{\prime} 6$ FAM fluorescein tag. Competitive EMSA assay was done with $50 \mathrm{nM}$ of 6-FAM labeled pLEE1 probe and unlabeled probes corresponding to $\mathrm{P}_{\mathrm{LEE} 1}, \mathrm{P}_{\text {kan }}$ (negative control) or $\mathrm{P}_{\text {nagB-nagE }}$ (positive control). The ratio "cold probe/labeled probe" was 10/1.

\section{DNAse Footprinting}

DNase I footprinting of free DNA and DNA-protein complexes was performed as described (El Qaidi et al., 2009; Graveline et al., 2011). The DNA fragment corresponding to the ler regulatory region (259 bp) was amplified using primers listed in Table EV2, alternately end labeled with ${ }^{32} \mathrm{P}(140,000 \mathrm{cpm}, 0.6 \mathrm{nM})$. Each endlabeled amplicon was subsequently incubated in a total volume of $80 \mu \mathrm{l}$ in binding buffer (25 mM Hepes ( $\mathrm{pH} 8.0), 100 \mathrm{mM}$ $\mathrm{K}$ glutamate ( $\mathrm{pH} 8.0), 0.5 \mathrm{mg} / \mathrm{ml} \mathrm{BSA})$. After incubation with purified NagC for $10 \mathrm{~min}$ at room temperature, $2 \mu \mathrm{l}$ of DNase I ( $1.3 \mathrm{U} / \mathrm{ml}$; New England BioLabs) containing $5 \mathrm{mM} \mathrm{CaCl}_{2}$ and $25 \mathrm{mM} \mathrm{MgCl} 2$ was added for $5 \mathrm{~min}$. The reaction was stopped by the addition of $150 \mu \mathrm{L}$ of phenol/chloroform/isoamyl alcohol and $350 \mu \mathrm{l}$ of stop buffer (0.5 M Na acetate pH 5.0, 2.5 mM EDTA, $10 \mu \mathrm{g} / \mathrm{ml}$ Salmon sperm DNA) to each sample. DNA fragments were precipitated in ethanol, and amounts with equivalent $\mathrm{cpm}$ $\left(5.10^{4}\right)$ from each reaction were loaded onto $6 \%$ polyacrylamide$7 \mathrm{M}$ urea gels. Maxam-Gilbert $\mathrm{A}+\mathrm{G}$ reactions were carried out on the appropriate 32 P-labeled DNA fragments, and the products loaded alongside the DNase I footprinting reaction mixtures. The gels were dried and analyzed by autoradiography. A control footprinting experiment realized with nagE-nagB regulatory region and with NagC (Figure EV5).

\section{Mice Infection}

$\mathrm{BALB} / \mathrm{c}$ mice were purchased from Janvier Labs (Le-Genest-StIsle, France). Sets of 5 mice aged 5 weeks were given drinking water containing streptomycin sulfate $(5 \mathrm{~g} / \mathrm{l})$ throughout the experiment. On day 1 following the addition of streptomycin, each mouse was infected intragastrically with $100 \mu \mathrm{l}$ of a mix containing $10^{7}$ each of EDL933 $\mathrm{Sm}^{\mathrm{R}}$ and EDL933 $\mathrm{Sm}^{\mathrm{R}} \Delta$ nagC strains. Mice treated with $B$. thetaiotaomicron were gavaged daily with $5 \times 10^{9}$ cells of $B$. thetaiotaomicron strain VPI-5482, starting from day 1 before EHEC infection to day 7. At indicated time points, fecal or tissue samples were collected, homogenized in PBS and subsequently diluted before plating on LB-streptomycin agar plates and LB-streptomycin-kanamycin agar plates. Output ratios were calculated for each time point and competitive indices were obtained by dividing the output ratio by the input ratio. A One-way ANOVA was performed to determine the significance. For NANA and NAG quantification in intestinal contents, see EV Methods.

\section{Ethics Statement}

All animal experiments were reviewed and approved by the Auvergne Committee for Animal Experimentation (C2E2A). All procedures were carried out according to the European directives for the protection of animals used for scientific purposes, $2010 / 63 / \mathrm{EU}$, and to the guidelines of the local ethics committee.

\section{RESULTS}

\section{NANA and NAG Inhibit EHEC 0157:H7 Adhesion to Epithelial Cells by Repressing LEE Genes}

The effect of NANA and NAG on EHEC adhesion was examined by measuring the ability of the EHEC O157:H7 EDL933 strain to adhere to cultured epithelial cells in the presence or absence of NANA or NAG. Our data showed that EDL933 adhesion to HeLa cells was significantly decreased by $40 \pm 21$ or $23 \pm 11 \%$ in presence of $1 \mathrm{mM} \mathrm{NAG}$ or NANA, respectively (Figure 1A; Figure EV1). At $0.1 \mathrm{mM}$, only NAG significantly decreased the number of cell-attached bacteria ( $53 \pm 22 \%)$.

The adhesion of EHEC O157:H7 to HeLa cells is mainly driven by the production of a T3SS, as previously demonstrated (Branchu et al., 2014) and as verified using the $\Delta e s c N$ strain which is defective in the production of the T3SS (Figure EV1). Thus, we investigated the effect of NANA and NAG on LEE gene expression. As a first step, strain EDL933 carrying a $\mathrm{P}_{\mathrm{LEE} 1}$ :lacZ fusion was cultured in the presence of different concentrations of the sugars. The expression of LEE1 was significantly repressed in the presence of either $1 \mathrm{mM}$ NANA or 0.1 and $1 \mathrm{mM} \mathrm{NAG}$ (Figure 1B), but not at $0.01 \mathrm{mM}$ of either sugar. Next, we examined the expression of genes from the five LEE operons and observed that expression of ler (LEE1), sepZ (LEE2), escV (LEE3), espB (LEE4), tir, and eae (LEE5) was significantly lower in presence of $1 \mathrm{mM}$ NANA or NAG (Figure 1C). Consistent with the decreased expression of the LEE4 gene, the secretion of the effector EspB, encoded by the LEE4, was dose dependently reduced when EDL933 was incubated with 0.1 and $1 \mathrm{mM}$ of 


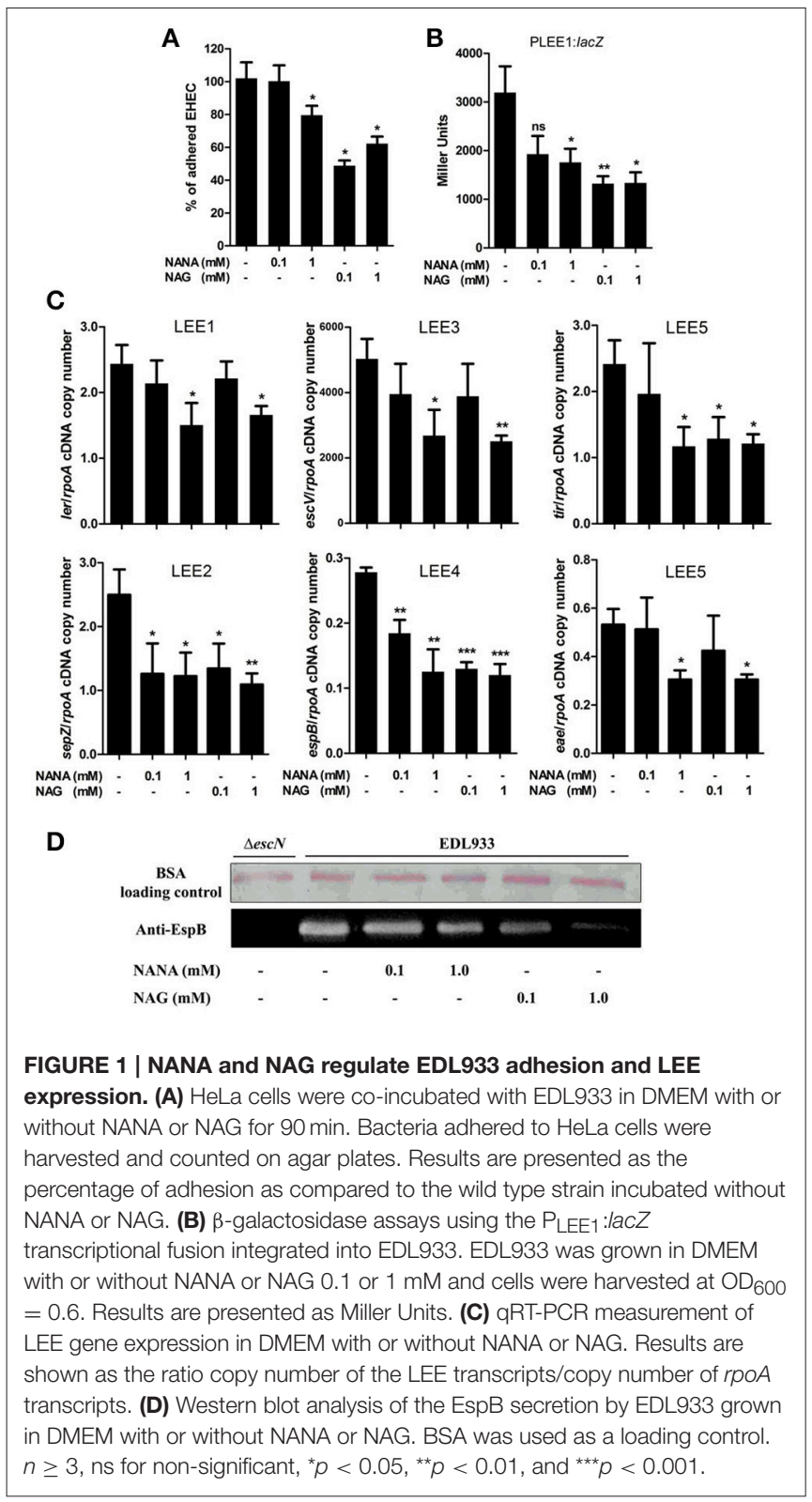

either sugar (Figure 1D). Taken together, these data indicate that NANA and NAG inhibit the adhesion of EDL933 to epithelial cells and repress T3SS encoding genes. In addition no significant difference was observed upon addition of other mucin sugars, such as mannose, galactose, $\mathrm{N}$-acetylgalactosamine and glucuronate on the expression of ler (Figure EV2).

\section{Repression of LEE Gene Transcription by NANA and NAG Is NagC-Dependent}

Activation of the metabolic pathways required for the catabolism of NANA and NAG influence the activity of two transcriptional regulators, NanR and $\mathrm{NagC}$ (Figure 2A). Intracellular NANA inactivates NanR while NAG-6P derived from the catabolic conversion of both NANA and NAG inactivates NagC. To evaluate the role of NanR and NagC on LEE1 promoter activity, the $\mathrm{P}_{\mathrm{LEE} 1}:$ lacZ fusion was introduced into $\Delta n a n R$ and $\Delta n a g C$ mutants. The nagC deletion led to a significant decrease of the activity of $\mathrm{P}_{\mathrm{LEE} 1}$ whereas the deletion of nanR had no significant effect (Figure 2B). Moreover, the addition of NANA or NAG still repressed the activity of $\mathrm{P}_{\mathrm{LEE} 1}$ in the $\Delta$ nanR mutant whereas it did not in the $\triangle$ nagC mutant. These results indicate that LEE1 repression by NANA and NAG was NagCdependent but NanR-independent. We further demonstrated that a $n a g C$ deletion also impairs the expression of escV, $\operatorname{sep} Z$, $\operatorname{esp} B$, tir, and eae genes with a fold change ranging from -2.9 to -5.5 (Figure 2C), as well as secretion of EspB (Figure 2D). Consequently, the $\triangle$ nagC mutant adhered less to epithelial cells, using HeLa and the two human intestinal cell lines HCT8 and HCT-116 (Figure 2E; Figures EV1, EV3). Importantly, LEE gene expression, EspB secretion and adhesion levels were restored to a wild-type status in a $\triangle n a g C$-complemented strain. Additionally, we assessed the potential involvement of $\mathrm{NagC}$ in the transcription of other genes encoding adhesins in EDL933. Neither fliC (flagellin), $y c b Q$ (fimbriae) (Samadder et al., 2009), $h c p A$ (hemorrhagic coli pilus), espP (autotransporter), lpfA (long polar fimbriae), csgA (curli) nor $p g a B$ (poly- $\beta-1,6-$ $\mathrm{N}$-acetyl-D-glucosamine) were differentially expressed between wild type and $\triangle n a g C$ strains (Supplementary Figure EV4). Since NagC does not affect the synthesis of these adhesins, it suggests that the defect in cell adhesion observed with the $n a g C$ mutant is mainly driven by a reduced production of the T3SS.

\section{NagC Interacts with LEE1 Promoter Region In vitro}

Using a NagC consensus DNA binding site generated from seven $\mathrm{NagC}$ binding sequences, we identified a single putative NagC binding site in the LEE1 promoter region (5'-GTATTTTACACATTAGAAAAAAG-3') located at a position that overlaps the -10 box of the distal promoter (Figure 3A). The binding of $\mathrm{NagC}$ to the LEE1 promoter was first investigated by EMSA that showed that NagC forms a specific low-mobility complex with the LEE1 promoter as previously observed with $\mathrm{NagC}$ interacting with type 1 fimbriae fim intergenic region upstream of the fimB promoter (Sohanpal et al., 2004). Competition EMSA using an unrelated probe $\left(\mathrm{P}_{\text {kan }}\right)$ demonstrated that NagC binding to the LEE1 promoter is specific (Figure 3B). Further, DNase footprinting experiments confirmed that $\mathrm{NagC}$ bound to the predicted $\mathrm{NagC}$ binding site (Figure 3C). Consistent with the expected specificity, a single base substitution at position 18 of the putative NagC binding site prevented DNAse protection by NagC (Figure 3D). An additional DNase footprinting control experiment using nagBE intergenic region as expected showed clear protection zones indicating the functional activity of $\mathrm{NagC}$ (Figure EV5). These findings demonstrate that NagC interacts with the LEE1 promoter region in a sequence specific manner. Interestingly, the NagC-binding sequence in the promoter of ler is conserved among other EHEC O157:H7 strains and this is correlated with ler repression in the presence of $1 \mathrm{mM}$ of NAG (Figure EV6). EHEC, EPEC, 

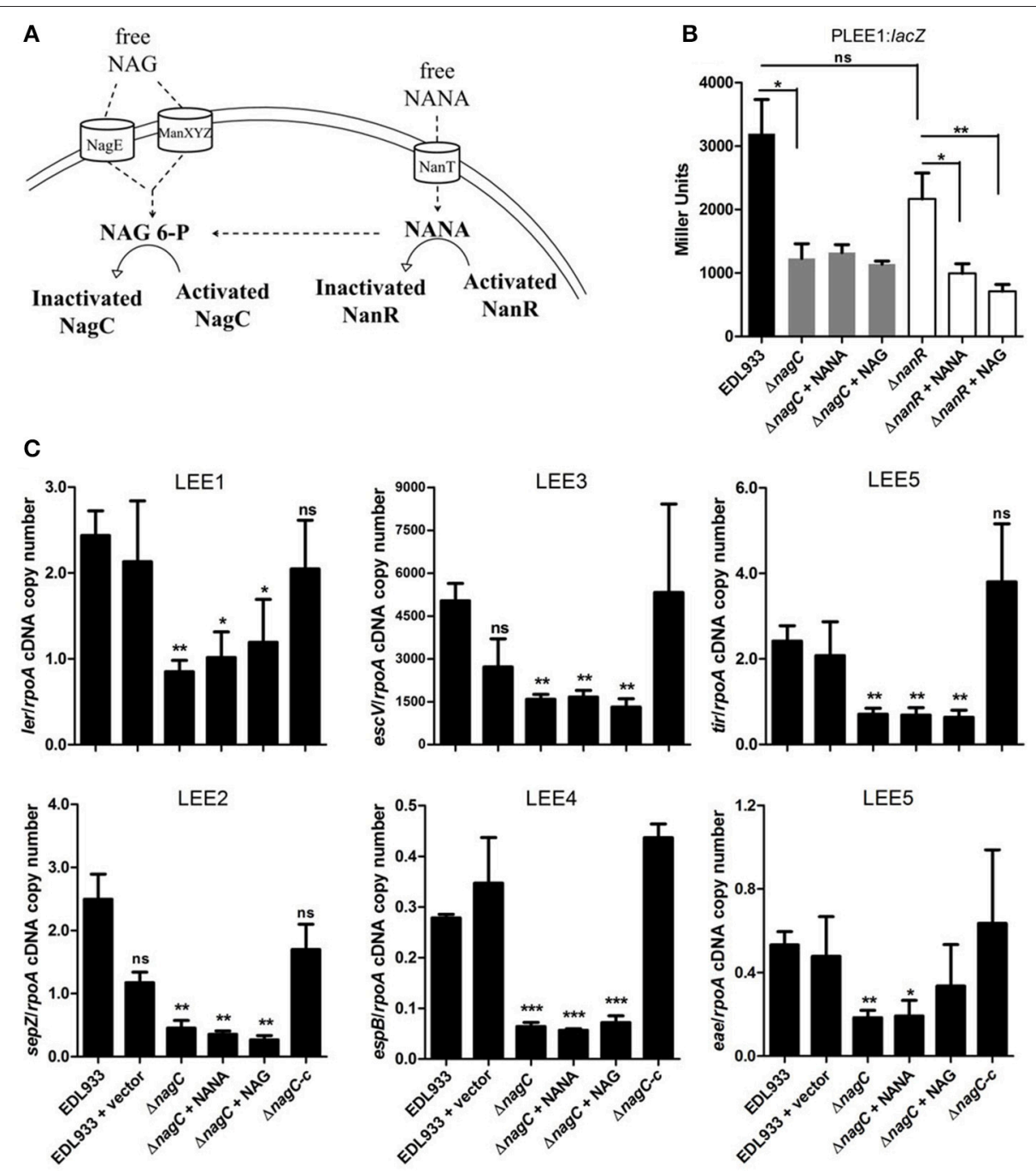

D
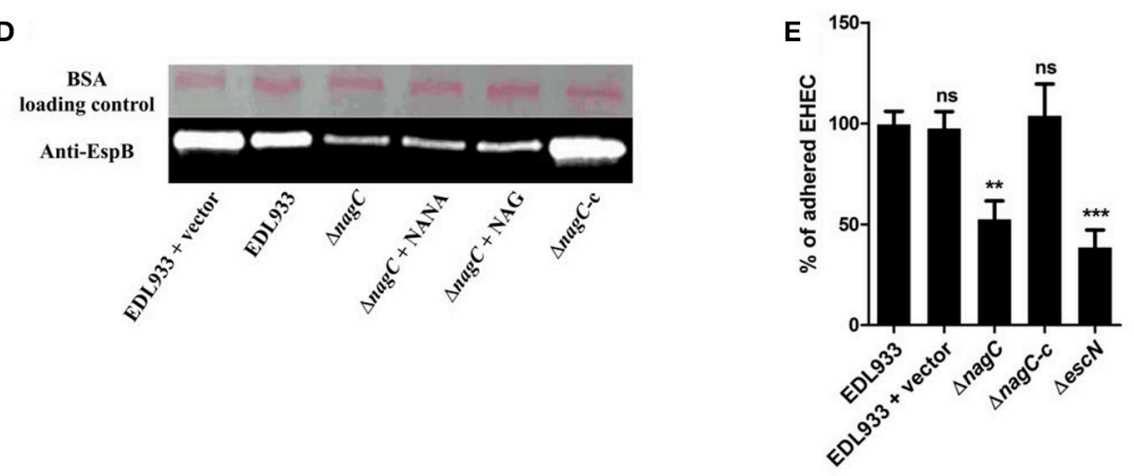

FIGURE 2 | NagC is a transcriptional activator of the LEE genes. (A) Schematic representation of the influence of NANA and NAG on the activity of the transcriptional regulators NagC and NanR. (B) $\beta$-galactosidase assays using the $P_{L E E 1}$ :lacZ transcriptional fusion integrated into EDL933 or the isogenic mutants $\triangle$ nagC and $\triangle$ nanR. The strains were grown in DMEM with or without NANA or NAG at $1 \mathrm{mM}$ and harvested at $\mathrm{OD}_{600}=0.6$. Results are presented as Miller Units. (C) qRT-PCR measurement of LEE gene expression. EDL933, the isogenic mutant $\triangle$ nagC and the complemented strain $\triangle$ nagC-c were grown in DMEM with or without NANA or NAG at $1 \mathrm{mM}$. Results are shown as the ratio copy number of the LEE transcripts/copy number of rpoA transcripts. (D) Western blot analysis of the EspB secretion by EDL933, the isogenic mutant $\triangle$ nagC and the complement grown in DMEM with or without NANA or NAG at 1 mM. BSA was used as a loading control. (E) HeLa cells were co-incubated for 90 min with either the wild type EDL933 strain, $\triangle$ nagC mutant, the $\triangle$ nagC complemented strain or the $\triangle$ escN mutant. Adhered bacteria were harvested and counted on agar plates. Results are presented as the percentage of adhered cells compared to the wild type strain EDL933. $n \geq 3$, ns for non-significant, ${ }^{*} p<0.05,{ }^{* *} p<0.01$, and ${ }^{* * *} p<0.001$. 
or C. rodentium strains with degenerated $\mathrm{NagC}$ binding site in the LEE1 promoter region were insensitive to NAG exposure.

\section{Mucin-Derived Sugars Sensing by NagC Is Important for Successful Colonization in Mice}

To assess if NagC regulates the gut colonization process, we co-infected mice with an equal mixture of wild-type EDL933 and the $\Delta$ nagC mutant and followed the outcome of each strain overtime. We observed a marked increase in the wildtype strain over the $\Delta$ nagC mutant in the feces at days 6 and 8 post-infection (competitive index (CI) of $13 \pm 5$ and $20 \pm 6$, respectively), as well as in the cecal content at day 8 (CI of $270 \pm$ 76) (Figures 4A,B). The competitive advantage of the wild-type strain was also recorded for bacteria adhering to cecal and colonic mucosa (Figure EV7). These data demonstrate that the deletion of nagC greatly impaired the ability of EDL933 to colonize the intestinal tract of mice.

We next sought to determine if the concentrations of NANA and NAG may alter EDL933 fitness in vivo through the modulation of NagC activity. For that, the drinking water of WT/ $\Delta$ nagC-infected mice was supplemented with purified NANA or NAG. Supplementation led to increased sugar concentrations in the cecal content of uninfected mice but not in the cecal content of infected mice (Figure 4C). Interestingly, NANA and NAG concentrations also significantly decreased in supplemented mice upon infection, with fold-change of 4.9- and 1.9, respectively, suggesting that EDL933 consumes NANA and NAG in the intestine of mice. In these conditions, NAG supplementation significantly decreased the competitive advantage of the wild-type strain over the $\triangle$ nagC mutant by a factor 7.1 (Figure 4B). Co-infected mice were also subjected to a daily gavage with $B$. thetaiotaomicron to see if the behavior of EHEC is modulated by the population level of a mucin degrading bacterium. Gavage of mice did not change NANA concentration in the gut of infected mice but led to a 1.9-fold increase of NAG concentration (Figure 5). Importantly, we observed that the competitive index between the wild-type strain and the $\triangle$ nagC mutant significantly dropped from 297 in control mice to 54 in B. thetaiotaomicron-treated animals (Figure 5). Overall, our findings indicate that NAG concentration in the intestine, derived notably from activity of mucin degrading commensals, such as B. thetaiotaomicron, affects the fitness of EHEC in vivo in a NagC-dependent manner.

\section{DISCUSSION}

This work demonstrates that the host mucin-derived sugars NAG and NANA inhibit the expression level of LEE genes in EHEC O157:H7 strain EDL933 and, consequently, inhibit the ability of the pathogen to adhere to epithelial cells in vitro. NAG and NANA are known to be used as carbon sources by commensal E. coli and EHEC O157:H7 in the gut (Fabich et al., 2008; Bertin et al., 2013, 2014; Conway and Cohen, 2015). Their catabolism induces transcriptional responses mediated by
NanR and/or NagC regulatory proteins with NanR controlling NANA catabolism and NagC controlling both NAG and galactose catabolism (Plumbridge, 1991; El Qaidi et al., 2009). The role of $\mathrm{NagC}$ as a repressor of the expression of $n a g E, n a g B$ and galP encoding the NAG PTS permease, the glucosamine-6-P deaminase, and the major galactose transporter respectively, was confirmed in EDL933 (Figure EV8). We also demonstrated that $\mathrm{NagC}$ controls the expression level of LEE genes in EDL933 through a direct activation of LEE1 gene transcription. NagC has been shown to modulate the expression of distinct adhesins in other E. coli strains. Indeed, NagC, together with NanR, activates the expression of $f i m B$ encoding for a recombinase required for the expression of the type I fimbriae in K-12 strain MG1655 (McClain et al., 1991; Sohanpal et al., 2004). This is not the case in EDL933 since this strain does not produce type 1 fimbriae due to a 16-bp deletion in the regulatory switch region of fimA (Vogeleer et al., 2015). While we observed no change in EDL933, a deletion of $n a g C$ has been shown to decrease expression of $\operatorname{csg} A B$ and csgDEFG genes and curli production in strain C600, though the mechanism remains unknown (Barnhart et al., 2006). Overall these observations suggest that $\mathrm{NagC}$ has served a common role as a regulator of adhesin expression during the evolution of several E. coli strains.

Most operons known to be controlled by NagC require two sites for NagC to function (nagE-B, chb, $\operatorname{glm} U, \operatorname{fim} B$ ) so that cooperative binding to two sites through DNA looping is necessary for regulation (Plumbridge, 1996; Sohanpal et al., 2004; El Qaidi et al., 2009; Brechemier-Baey et al., 2015). However, like the galP promoter, another target of NagC (El Qaidi et al., 2009), only one potential NagC operator is found in LEE1 promoter region of EHEC strain EDL933. Interestingly, this NagC sequence was conserved in other O157:H7 strains but not in other LEE encoding pathogens, such as EPEC E2348/69 or $C$. rodentium ICC168. Yet it is not clear how NagC activates the LEE1 promoter. As proposed by authors working on $\mathrm{fimB}$ and galP, NagC could contact RNA polymerase directly (or another regulatory protein bound closer to the LEE1 promoter region) to enhance transcription activation, or that the nucleoprotein complex that includes $\mathrm{NagC}$ and other regulators alters the DNA structure nearer the promoter in such a way as to facilitate transcription initiation (Sohanpal et al., 2004, 2007; El Qaidi and Plumbridge, 2008; El Qaidi et al., 2009).

By regulating genes involved in sugar catabolism and genes involved in T3SS production, NagC is likely to influence the behavior of EHEC during an infection. Indeed, we demonstrated by co-infection experiments that deletion of nagC strongly affect the fitness of EHEC in the digestive tract of infected mice. Moreover, the addition of NAG in the drinking water of infected mice reduced the competitive advantage of the wild type strain over the $\Delta$ nagC mutant. This suggests that intestinal concentration of NAG modulates $\mathrm{NagC}$ activation and therefore expression level of NagC-dependent genes, affecting the fitness of EHEC in vivo. In the gut, some commensal species expressing mucinolytic enzymes can degrade mucins from the outer layer of mucus and release free carbohydrates into the intestinal lumen (Xu et al., 2003; Elhenawy et al., 2014; Tailford et al., 2015). These sugars can then be consumed by members of the gut 
A
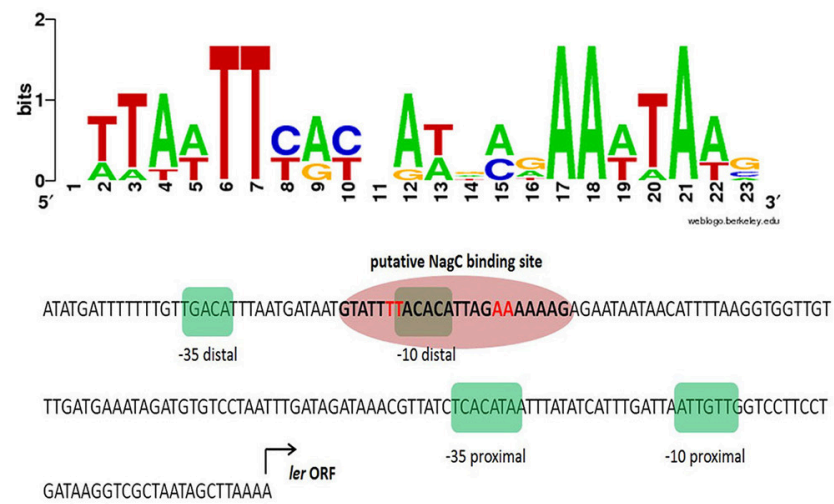

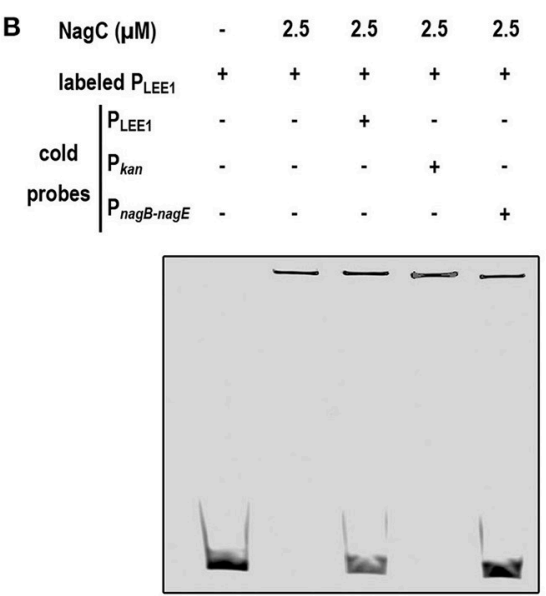

C

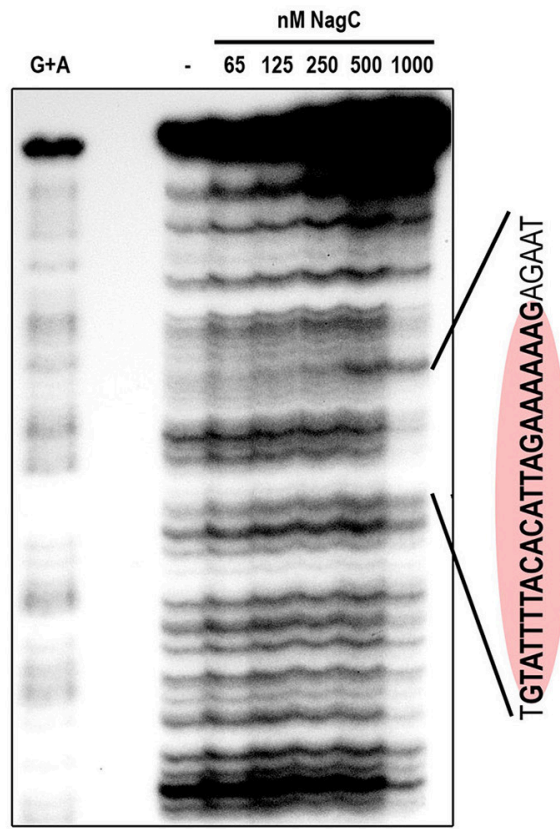

D

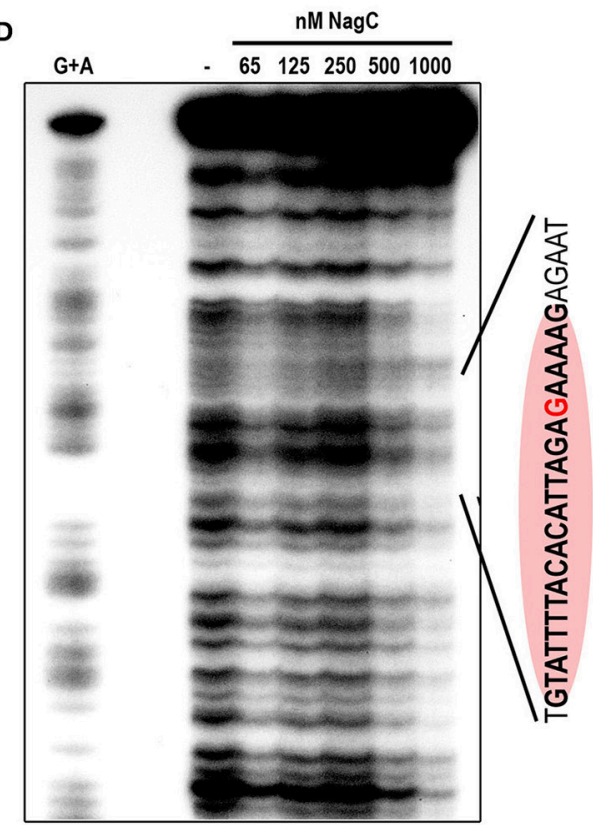

FIGURE 3 | NagC binds in vitro to the promoter of LEE1. (A) Predicted binding site generated by Weblogo from seven known NagC binding sequences and schematic representation of the regulatory region of LEE1. The -35 and -10 boxes of the proximal and the distal promoters are highlighted in green and the putative NagC binding site in red. (B) Competitive EMSA assays were performed using purified NagC (2.5 $\mu$ M) and a 6-FAM labeled PLEE1 probe (50 nM) and unlabeled probes corresponding to $\mathrm{P}_{\mathrm{LEE} 1}, \mathrm{P}_{\mathrm{kan}}$ (negative control) or $\mathrm{P}_{\text {nagB-nagE }}$ (positive control). (C) Footprinting experiment was performed with end-labeled PCR product of the native LEE1 regulatory region and purified NagC. The DNA sequence of the protected region is indicated and includes the NagC putative binding sequence (red ellipse). (D) Footprinting experiment was performed with end-labeled PCR product of the mutated LEE1 regulatory region. The base substitution (A $\rightarrow$ G) is indicated in red.

microbiota (Derrien et al., 2010). By affecting the concentration of free NAG and NANA available in the digestive tract, gut bacterial species expressing sialidase or $\mathrm{N}$-acetylglucosaminidase might therefore affect the fitness of EHEC through a modulation of NagC activity. Indeed, we showed that the metabolic activity of the mucin degrader $B$. thetaiotaomicron when gavaged to coinfected mice increased the concentration of NAG and reduced the competitive advantage of the wild-type strain over the nagC mutant. To our knowledge, no information is available on NAG and NANA concentration in human intestine. However, its concentration probably fluctuates within the gastrointestinal tract since there is high variations in terms of (i) abundance and types of mucins; (ii) patterns of O-glycosylation and (iii) bioavailability of carbohydrates (free or mucin-linked forms). In Figures 4, 5, the concentration of NANA and NAG in the cecal content of uninfected mouse was $0.27 \mathrm{mM}(500 \mathrm{nmol} / \mathrm{g})$ and $0.12 \mathrm{mM}$ (225 nmol/g), respectively. In another study, NANA and NAG were quantified in the bovine small intestine content to be 0.1 and $0.45 \mathrm{mM}$ (Bertin et al., 2013). Altogether, it gives information on physiological NAG and NANA concentrations in 
A

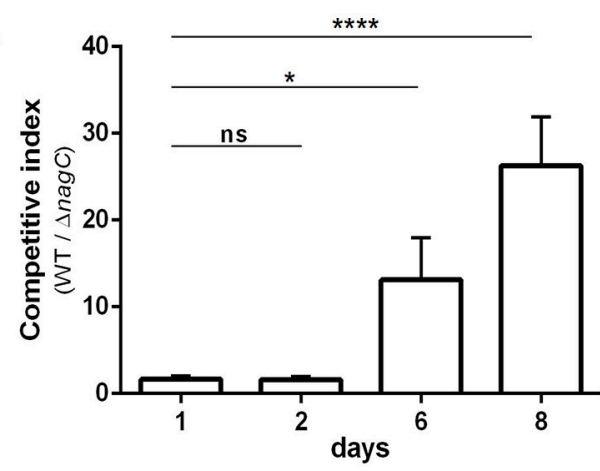

C

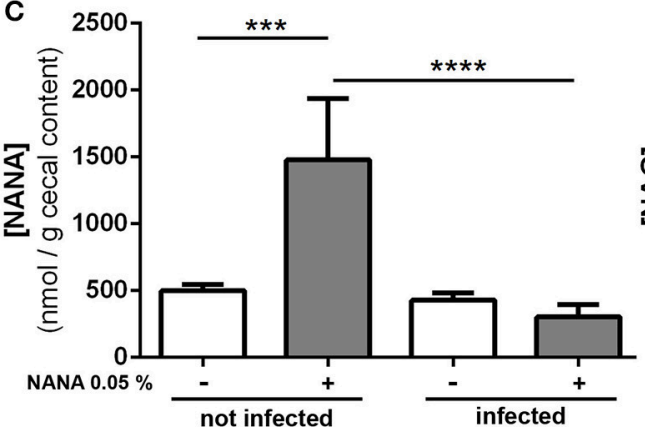

B
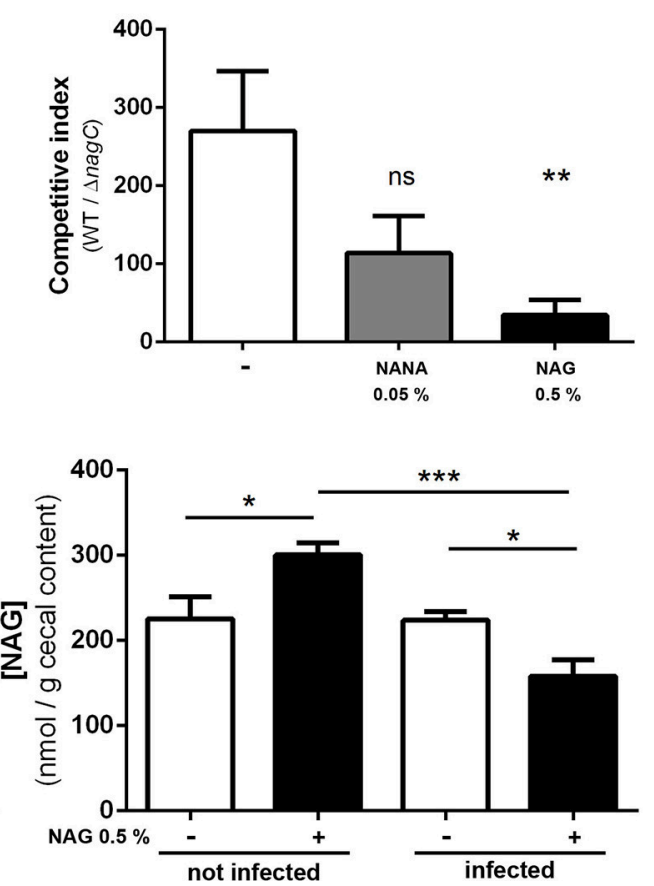

FIGURE 4 | The $\Delta$ nag $\mathbf{C}$ mutant is outcompeted by the wild type strain during mice infection. Streptomycin-treated BALBc mice were infected with a 1:1 mixture of wild type and $\triangle$ nagC EDL933 strains. (A) Wild type and $\Delta$ nagC strains were numerated from feces and competitive indices WT/ $\Delta$ nagC were calculated at indicated time points. (B) Competitive indices WT/ $\mathrm{B}$ nagC obtained at day 8 in the cecal contents of mice provided with water with or without NANA $0.05 \%$ or NAG 0.5\%. (C) Concentration of NANA and NAG in the cecal contents of non-infected mice or EHEC-infected mice provided with or without either NANA 0.05\% or NAG $0.5 \%$. ${ }^{*} p<0.05,{ }^{* *} p<0.01,{ }^{* * *} p<0.001$, and ${ }^{* * * *} p<0.0001$.

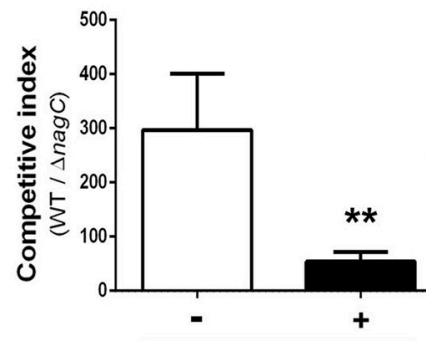

B. thetaiotaomicron

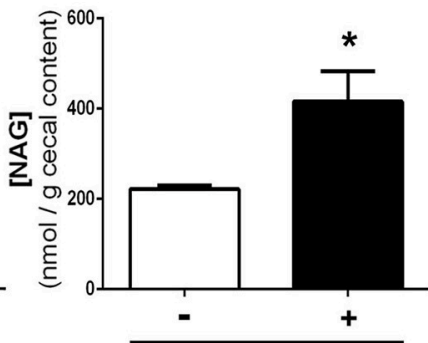

B. thetaiotaomicron

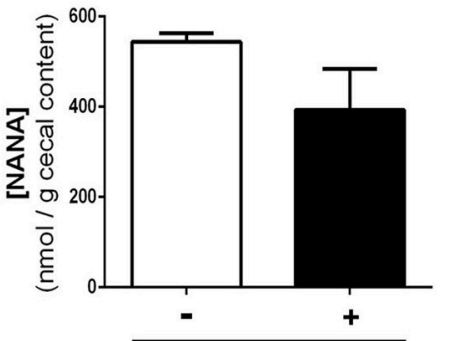

B. thetaiotaomicron

FIGURE 5 | Bacteroides thetaiotaomicron influences the outcome of wild type and $\mathbf{\Delta}$ nagC strains in co-infected mice. Streptomycin-treated BALBC were infected with a 1:1 mixture of wild type and $\triangle$ nagC EDL933 strains. Mice were gavaged or not daily with $5 \times 10^{9} \mathrm{~B}$. thetaiotaomicron cells starting 1 day before EHEC infection. Competitive indices WT/ $\triangle$ nagC and concentrations of NAG and NANA obtained at day 8 post-infection in the cecal contents of mice treated or not with $B$. thetaiotaomicron are shown. ${ }^{*} p<0.05$ and ${ }^{* *} p<0.01$.

digestive tracts and indicates that concentrations used in our in vitro studies were relevant.

If we determined that NagC is essential for the fitness of EHEC in the digestive tract of mice, we have no information about the NagC-regulated genes involved in fitness alteration. Fabich et al. demonstrated that a mutation in gene nagE encoding the NAG transporter, causes a colonization defect for EDL933 in infected mice, indicating that NAG is utilized by the pathogen in the digestive tract of mice. In contrast, a mutation in nanAT where nanT encodes the NANA transporter, has no impact on colonization efficiency (Fabich et al., 2008), indicating that NANA catabolism is not essential for a good colonization of mice gut. In contrast to nagE and nanAT mutants that are unable to internalize NAG and NANA respectively, nagC mutant is still able to uptake and catabolize both sugars since gene deletion leads to an upregulation of $n a g E$ and $n a g B A C D$ gene expression (Figure EV8). In addition to sugar catabolism and T3SS related genes, $\mathrm{NagC}$ also probably controls the expression of other genes in EHEC, that might be involved in EHEC fitness. One example is the gene z2210 (Figure EV9), which encodes a putative sulfatase 
that might be involved in mucus degradation since secreted mucins are heavily sulfated (Nieuw Amerongen et al., 1998). NagC of Vibrio fischeri was shown to facilitate colonization of the light organ of the squid Euprymna scolopes (Miyashiro et al., 2011). Sun Y et al. proposed that in V. fischeri NagC ability to regulate gene expression contributes to its overall fitness in environments that vary in levels of GlcNAc (Sun et al., 2015).

In many pathogens, relationship between metabolism and virulence has been determined (Wilharm and Heider, 2014). We propose that NagC is part of the regulatory circuit controlling the infectious process of EHEC by coordinating mucin-derived sugar metabolism and T3SS production (Figure EV10). At the level of the colonic intestine within the mucus there is a gradient of NANA and NAG due to their release from the intestinal mucin by mucinolytic bacteria, such as $B$. thetaiotaomicron (Derrien et al., 2010). When the concentration of NANA and/or NAG is high, their catabolism by E. coli O157:H7 produces high amount of intracellular NAG-6P which inactivates the transcriptional regulator NagC. In such case, the expression of NAG catabolic genes is induced while that of the LEE genes is reduced and thus adherence is prevented. This suggestion is supported by our observation that expression of LEE operons decreased after EDL933 growth in cecal content of gnotobiotic rats inoculated with human cecal content (Le Bihan et al., 2015). In reaching deeper mucus layer toward the intestinal epithelium, NANA and NAG are less found as free forms in the inner layer of mucus but are rather complexed to mucins (Derrien et al., 2010). In consequence, the amount of intracellular NAG-6P is low and the protein $\mathrm{NagC}$ is active allowing the repression of $n a g B, n a g E$ and galP and activation of the LEE genes and thus adherence is promoted. Such a mechanism could contribute to the relocation of the pathogen from the intestinal lumen to the surface of intestinal epithelial cells, as previously suggested by others (Kamada et al., 2012; Pacheco et al., 2012; Cameron and Sperandio, 2015).

In this study, we described a novel mechanism by which EHEC O157:H7 regulate the expression of its T3SS-encoding genes in response to sugars derived from intestinal mucin. The NAG-6P sensor NagC was shown to promote the adherence of EHEC O157:H7 to intestinal cells in vitro through a direct regulation of ler and to be an important regulator for the fitness

\section{REFERENCES}

Barnhart, M. M., Lynem, J., and Chapman, M. R. (2006). GlcNAc-6P levels modulate the expression of Curli fibers by Escherichia coli. J. Bacteriol. 188, 5212-5219. doi: 10.1128/JB.00234-06

Bertin, Y., Chaucheyras-Durand, F., Robbe-Masselot, C., Durand, A., de la Foye, A., Harel, J., et al. (2013). Carbohydrate utilization by enterohaemorrhagic Escherichia coli O157:H7 in bovine intestinal content. Environ. Microbiol. 15, 610-622. doi: 10.1111/1462-2920.12019

Bertin, Y., Deval, C., de la Foye, A., Masson, L., Gannon, V., Harel, J., et al. (2014). The gluconeogenesis pathway is involved in maintenance of enterohaemorrhagic Escherichia coli O157:H7 in bovine intestinal content. PLoS ONE 9:e98367. doi: 10.1371/journal.pone.0098367

Branchu, P., Matrat, S., Vareille, M., Garrivier, A., Durand, A., Crepin, S., et al. (2014). NsrR, GadE, and GadX interplay in repressing expression of the Escherichia coli O157:H7 LEE pathogenicity island in response to nitric oxide. PLoS Pathog. 10:e1003874. doi: 10.1371/journal.ppat.1003874 of EHEC in vivo. This work sheds further light on the link between the nutrient availability and EHEC O157:H7 adaptation and virulence gene expression.

\section{AUTHOR CONTRIBUTIONS}

Conceived and designed the experiments: GL, CM, JH, and GJ. Performed the experiments: GL, JS, PG, AG, FB, and GJ. Analyzed the data: GL, AB, APG, CM, JH, and GJ. Wrote the paper: GL, CM, JH, and GJ.

\section{FUNDING}

GL was supported by a scholarship from the Institut de recherche en santé publique de l'Université de Montréal-Fonds de recherche du Québec-santé (Project 40148). This work was also supported in part by the 61st Session de la Commission permanente de coopération franco-québécoise (Project 61. 116 to $\mathrm{CM}$ and $\mathrm{JH}$ ), by the Natural Sciences and Engineering Research Council of Canada (NSERC) (Strategic and Discovery Grants to JH, RGPIN STP 307430 and RGPIN-2015-05373, respectively) and by EADGENE, N FOOD-CT-2004-506416, Network of Excellence under the 6th Research Framework Program of the European Union (CM).

\section{ACKNOWLEDGMENTS}

We thank C. Del'Homme, E. Delmas, G. Lopes and J. Daniel for excellent technical assistance. We are grateful to Dr. J. Plumbridge (Institut de Biologie Physico-Chimique, Paris) her helpful comments and sharing information on NagC and help in identifying putative NagC binding DNA sequences and to Judith Kashul, for editing the manuscript.

\section{SUPPLEMENTARY MATERIAL}

The Supplementary Material for this article can be found online at: http://journal.frontiersin.org/article/10.3389/fcimb. 2017.00134/full\#supplementary-material

Brechemier-Baey, D., Dominguez-Ramirez, L., Oberto, J., and Plumbridge, J. (2015). Operator recognition by the ROK transcription factor family members, NagC and Mlc. Nucleic Acids Res. 43, 361-372. doi: 10.1093/nar/ gku1265

Cameron, E. A., and Sperandio, V. (2015). Frenemies: signaling and nutritional integration in pathogen-microbiota-host interactions. Cell Host Microb. 18, 275-284. doi: 10.1016/j.chom.2015.08.007

Chekabab, S. M., Jubelin, G., Dozois, C. M., and Harel, J. (2014). PhoB activates Escherichia coli $\mathrm{O} 157: \mathrm{H} 7$ virulence factors in response to inorganic phosphate limitation. PLoS ONE 9:e94285. doi: 10.1371/journal.pone.0094285

Conway, T., and Cohen, P. S. (2015). Commensal and pathogenic Escherichia coli metabolism in the gut. Microb. Spectr. 3:MBP-0006-2014. doi: 10.1128/microbiolspec.MBP-0006-2014

Deng, W., Puente, J. L., Gruenheid, S., Li, Y., Vallance, B. A., Vazquez, A., et al. (2004). Dissecting virulence: systematic and functional analyses of a pathogenicity island. Proc. Natl. Acad. Sci. U.S.A. 101, 3597-3602. doi: 10.1073/pnas.0400326101 
Derrien, M., van Passel, M. W., van de Bovenkamp, J. H., Schipper, R. G., de Vos, W. M., and Dekker, J. (2010). Mucin-bacterial interactions in the human oral cavity and digestive tract. Gut. Microb. 1, 254-268. doi: 10.4161/gmic.1.4.12778

Elhenawy, W., Debelyy, M. O., and Feldman, M. F. (2014). Preferential packing of acidic glycosidases and proteases into Bacteroides outer membrane vesicles. mBio 5, e00909-14. doi: 10.1128/mBio.00909-14

El Qaidi, S., Allemand, F., Oberto, J., and Plumbridge, J. (2009). Repression of galP, the galactose transporter in Escherichia coli, requires the specific regulator of N-acetylglucosamine metabolism. Mol. Microbiol. 71, 146-157. doi: 10.1111/j.1365-2958.2008.06515.x

El Qaidi, S., and Plumbridge, J. (2008). Switching control of expression of ptsG from the Mlc regulon to the NagC regulon. J. Bacteriol. 190, 4677-4686. doi: 10.1128/JB.00315-08

Fabich, A. J., Jones, S. A., Chowdhury, F. Z., Cernosek, A., Anderson, A., Smalley, D., et al. (2008). Comparison of carbon nutrition for pathogenic and commensal Escherichia coli strains in the mouse intestine. Infect. Immun. 76, 1143-1152. doi: 10.1128/IAI.01386-07

Graveline, R., Mourez, M., Hancock, M. A., Martin, C., Boisclair, S., and Harel, J. (2011). Lrp-DNA complex stability determines the level of ON cells in type P fimbriae phase variation. Mol. Microbiol. 81, 1286-1299. doi: 10.1111/j.1365-2958.2011.07761.x

Kamada, N., Kim, Y. G., Sham, H. P., Vallance, B. A., Puente, J. L., Martens, E. C., et al. (2012). Regulated virulence controls the ability of a pathogen to compete with the gut microbiota. Science 336, 1325-1329. doi: 10.1126/science.1222195

Kaper, J. B., Nataro, J. P., and Mobley, H. L. (2004). Pathogenic Escherichia coli. Nat. Rev. Microbiol. 2, 123-140. doi: 10.1038/nrmicro818

Kendall, M. M., Gruber, C. C., Parker, C. T., and Sperandio, V. (2012). Ethanolamine controls expression of genes encoding components involved in interkingdom signaling and virulence in enterohemorrhagic Escherichia coli O157:H7. mBio 3:e0050-12. doi: 10.1128/mBio.00050-12

Le Bihan, G., Jubelin, G., Garneau, P., Bernalier-Donadille, A., Martin, C., Beaudry, F., et al. (2015). Transcriptome analysis of Escherichia coli O157:H7 grown in vitro in the sterile-filtrated cecal content of human gut microbiota associated rats reveals an adaptive expression of metabolic and virulence genes. Microb. Infect. 17, 23-33. doi: 10.1016/j.micinf.2014.09.008

Leedle, J. A., and Hespell, R. B. (1980). Differential carbohydrate media and anaerobic replica plating techniques in delineating carbohydrate-utilizing subgroups in rumen bacterial populations. Appl. Environ. Microbiol. 39, 709-719.

McClain, M. S., Blomfield, I. C., and Eisenstein, B. I. (1991). Roles of fimB and fimE in site-specific DNA inversion associated with phase variation of type 1 fimbriae in Escherichia coli. J. Bacteriol. 173, 5308-5314.

McGuckin, M. A., Linden, S. K., Sutton, P., and Florin, T. H. (2011). Mucin dynamics and enteric pathogens. Nat. Rev. Microbiol. 9, 265-278. doi: $10.1038 /$ nrmicro 2538

Miller, J. H. (1972). Experiments in Molecular Genetics. Cold Spring Harbor, NY: Cold Spring Harbor Laboratory.

Miyashiro, T., Klein, W., Oehlert, D., Cao, X., Schwartzman, J., and Ruby, E. G. (2011). The N-acetyl-D-glucosamine repressor NagC of Vibrio fischeri facilitates colonization of Euprymna scolopes. Mol. Microbiol. 82, 894-903. doi: 10.1111/j.1365-2958.2011.07858.x

Nakanishi, N., Tashiro, K., Kuhara, S., Hayashi, T., Sugimoto, N., and Tobe, T. (2009). Regulation of virulence by butyrate sensing in enterohaemorrhagic Escherichia coli. Microbiology 155, 521-530. doi: 10.1099/mic.0. 023499-0

Ng, K. M., Ferreyra, J. A., Higginbottom, S. K., Lynch, J. B., Kashyap, P. C., Gopinath, S., et al. (2013). Microbiota-liberated host sugars facilitate post-antibiotic expansion of enteric pathogens. Nature 502, 96-99. doi: 10.1038/nature12503

Nieuw Amerongen, A. V., Bolscher, J. G., Bloemena, E., and Veerman, E. C. (1998). Sulfomucins in the human body. Biol. Chem. 379, 1-18.

O’Brien, A. O., Lively, T. A., Chen, M. E., Rothman, S. W., and Formal, S. B. (1983). Escherichia coli O157:H7 strains associated with haemorrhagic colitis in the United States produce a Shigella dysenteriae 1 (SHIGA) like cytotoxin. Lancet 1, 702 .

Pacheco, A. R., Curtis, M. M., Ritchie, J. M., Munera, D., Waldor, M. K., Moreira, C. G., et al. (2012). Fucose sensing regulates bacterial intestinal colonization. Nature 492, 113-117. doi: 10.1038/nature11623
Plumbridge, J. (1996). How to achieve constitutive expression of a gene within an inducible operon: the example of the nagC gene of Escherichia coli. J. Bacteriol. 178, 2629-2636.

Plumbridge, J. A. (1991). Repression and induction of the nag regulon of Escherichia coli K-12: the roles of nagC and nagA in maintenance of the uninduced state. Mol. Microbiol. 5, 2053-2062.

Plumbridge, J., and Kolb, A. (1991). CAP and Nag repressor binding to the regulatory regions of the nagE-B and manX genes of Escherichia coli. J. Mol. Biol. 217, 661-679.

Porter, M. E., Mitchell, P., Free, A., Smith, D. G., and Gally, D. L. (2005). The LEE1 promoters from both enteropathogenic and enterohemorrhagic Escherichia coli can be activated by PerC-like proteins from either organism. J. Bacteriol. 187, 458-472. doi: 10.1128/Jb.187.2.458-472.2005

Samadder, P., Xicohtencatl-Cortes, J., Saldana, Z., Jordan, D., Tarr, P. I., Kaper, J. B., et al. (2009). The Escherichia coli ycbQRST operon encodes fimbriae with laminin-binding and epithelial cell adherence properties in Shiga-toxigenic E. coli O157:H7. Environ. Microbiol. 11, 1815-1826. doi: 10.1111/j.1462-2920.2009.01906.x

Sircili, M. P., Walters, M., Trabulsi, L. R., and Sperandio, V. (2004). Modulation of enteropathogenic Escherichia coli virulence by quorum sensing. Infect. Immun. 72, 2329-2337. doi: 10.1128/IAI.72.4.2329-2337.2004

Sohanpal, B. K., El-Labany, S., Lahooti, M., Plumbridge, J. A., and Blomfield, I. C. (2004). Integrated regulatory responses of fimB to N-acetylneuraminic (sialic) acid and GlcNAc in Escherichia coli K-12. Proc. Natl. Acad. Sci. U.S.A. 101, 16322-16327. doi: 10.1073/pnas.0405821101

Sohanpal, B. K., Friar, S., Roobol, J., Plumbridge, J. A., and Blomfield, I. C. (2007) Multiple co-regulatory elements and IHF are necessary for the control of fimB expression in response to sialic acid and $\mathrm{N}$-acetylglucosamine in Escherichia coli K-12. Mol. Microbiol. 63, 1223-1236. doi: 10.1111/j.1365-2958.2006.05583.x

Sperandio, V., Li, C. C., and Kaper, J. B. (2002). Quorum-sensing Escherichia coli regulator A: a regulator of the LysR family involved in the regulation of the locus of enterocyte effacement pathogenicity island in enterohemorrhagic $E$. coli. Infect. Immun. 70, 3085-3093. doi: 10.1128/IAI.70.6.3085-3093.2002

Sun, Y., Verma, S. C., Bogale, H., and Miyashiro, T. (2015). NagC represses Nacetyl-glucosamine utilization genes in Vibrio fischeri within the light organ of Euprymna scolopes. Front. Microbiol. 6:741. doi: 10.3389/fmicb.2015.00741

Tailford, L. E., Crost, E. H., Kavanaugh, D., and Juge, N. (2015). Mucin glycan foraging in the human gut microbiome. Front. Genet. 6:81. doi: 10.3389/fgene.2015.00081

Vogeleer, P., Tremblay, Y. D., Jubelin, G., Jacques, M., and Harel, J. (2015). Biofilm-forming abilities of Shiga toxin-producing Escherichia coli isolates associated with human infections. Appl. Environ. Microbiol. 82, 1448-1458. doi: 10.1128/aem.02983-15

Walters, M., and Sperandio, V. (2006). Autoinducer 3 and epinephrine signaling in the kinetics of locus of enterocyte effacement gene expression in enterohemorrhagic Escherichia coli. Infect. Immun. 74, 5445-5455. doi: 10.1128/IAI.00099-06

Wilharm, G., and Heider, C. (2014). Interrelationship between type three secretion system and metabolism in pathogenic bacteria. Front. Cell. Infect. Microbiol. 4:150. doi: $10.3389 /$ fcimb.2014.00150

Xu, J., Bjursell, M. K., Himrod, J., Deng, S., Carmichael, L. K., Chiang, H. C., et al. (2003). A genomic view of the human-Bacteroides thetaiotaomicron symbiosis. Science 299, 2074-2076. doi: 10.1126/science.1080029

Yang, B., Feng, L., Wang, F., and Wang, L. (2015). Enterohemorrhagic Escherichia coli senses low biotin status in the large intestine for colonization and infection. Nat. Commun. 6, 6592. doi: 10.1038/ncomms7592

Conflict of Interest Statement: The authors declare that the research was conducted in the absence of any commercial or financial relationships that could be construed as a potential conflict of interest.

Copyright (c) 2017 Le Bihan, Sicard, Garneau, Bernalier-Donadille, Gobert, Garrivier, Martin, Hay, Beaudry, Harel and Jubelin. This is an open-access article distributed under the terms of the Creative Commons Attribution License (CC BY). The use, distribution or reproduction in other forums is permitted, provided the original author(s) or licensor are credited and that the original publication in this journal is cited, in accordance with accepted academic practice. No use, distribution or reproduction is permitted which does not comply with these terms. 